\title{
Janus Particles in a Nonpolar Solvent (Supporting Information)
}

\author{
Joohyung Lee, ${ }^{\sharp}$ Benjamin A. Yezer ${ }^{\S}$ Dennis C. Prieve ${ }^{\S}$ and Sven Holger Behrens ${ }^{*}$
}

${ }^{¥}$ School of Chemical and Biomolecular Engineering, Georgia Institute of Technology, 311 Ferst Drive NW, Atlanta, GA 30332, USA

${ }^{\S}$ Center for Complex Fluids Engineering and Department of Chemical Engineering, Carnegie Mellon University, Pittsburgh, PA 15213, USA

\section{Corresponding Author:}

*Sven H. Behrens (하ehrens@gatech.edu)

This material includes the following experimental details:

S1. Scanning Electron Microscope (SEM) Imaging

S2. Dynamic Light Scattering (DLS) and 3D Cross Correlation DLS (3DDLS)

S3. Interfacial Tension Measurement

S4. Conductivity Measurement

S5. Electrochemical Impedance Spectroscopy (EIS)

S6. Karl Fischer Titration 


\section{S1. Scanning Electron Microscope (SEM) Imaging}

The morphology of the synthesized particles was characterized by scanning electron microscope (SEM), using Ultra-60 Field Emission SEM (Carl Zeiss AG). A small volume of diluted particle dispersion (20 ppm) was pipetted on the glass substrate and dried under ambient condition. The particles deposited on the glass slide were sputter-coated with small gold/palladium particles before characterization, to prevent charging. An accelerating voltage of $5 \mathrm{kV}$ was used. Figure $\mathrm{S} 1$ shows a representative SEM image of (A) the hydrophilic spherical particles (synthesized in the first seeded emulsion polymerization step) and the Janus snowman particles.
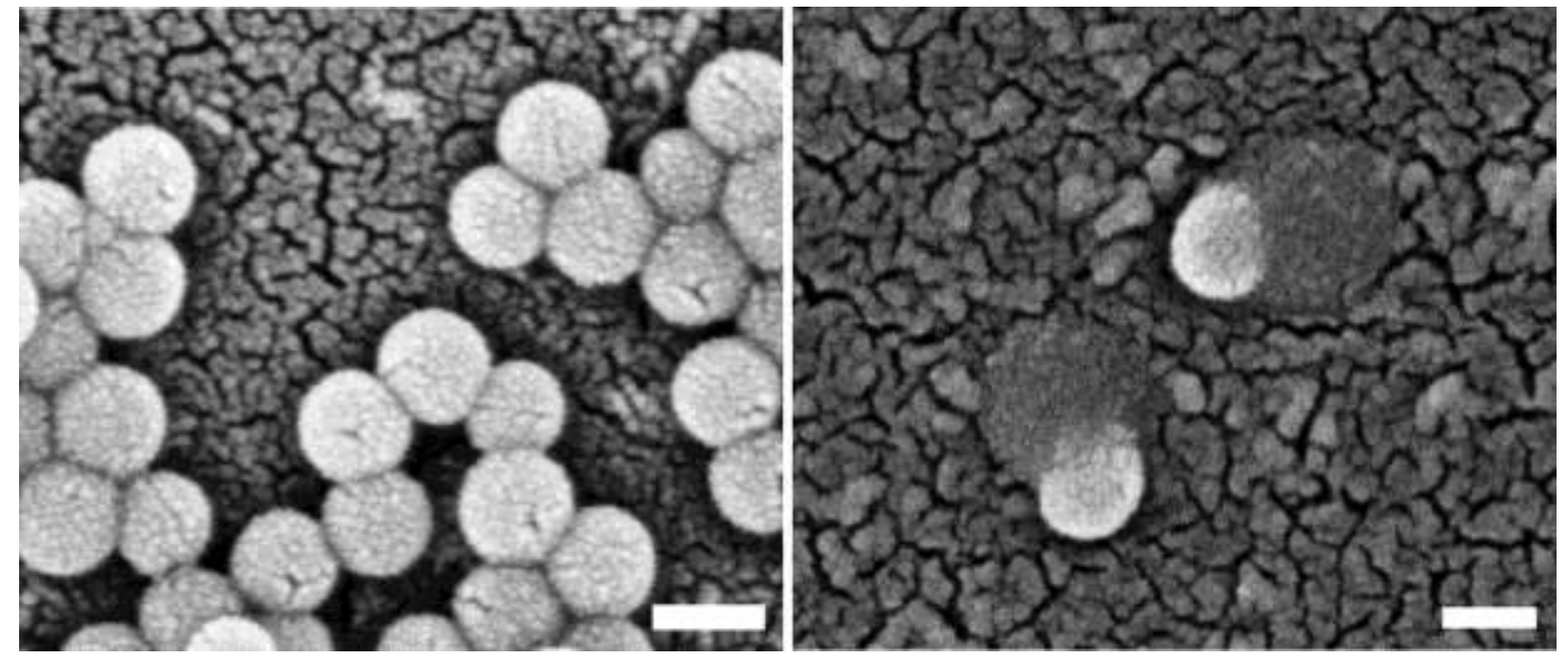

Figure S1. (A) SEM image of the hydrophilic spherical bulbs, synthesized in the first seeded emulsion polymerization step. (B) SEM image of the snowman particles, consisting of the hydrophilic bulb and a hydrophobic bulb synthesized in the second polymerization step. The patterns in the background are caused by sputter-coating with small gold/palladium particles. The scale bar represents 100nm. 


\section{S2. Dynamic Light Scattering (DLS) and 3D Cross Correlation DLS (3DDLS)}

For characterizing the solvodynamic diameter of oil-borne particles in nonpolar dispersions with low particle concentration ( $7 \mathrm{ppm}$, where the oil continuous phase looks almost clear), we used an ALV DLS/SLS-5022F (ALV-Laser GmBH) standard goniometer system. A second order cumulant fit to the intensity autocorrelation function was used to obtain the solvodynamic diameter of particles in the diluted state. For dispersions with high particle concentration $(300,1500$, and 8600 ppm, where the oil continuous phase looks slightly turbid to completely milky), we employed 3D-DLS technique to suppress the undesired contribution of multiply scattered light, using a 3D cross-correlation setup manufactured by LS Instruments AG, Switzerland. The 3D technique applies two simultaneous DLS experiments on the same scattering volume and at the same scattering vector and cross-correlates the respective scattering intensities in order to extract only the information from single scattering events common to both scattering signals. The laser wavelength is $\lambda=632.8 \mathrm{~nm}$ and scattered light is collected by two avalanche photodiode detectors mounted on a rotating goniometer. The measurements were taken at the scattering angles from $20^{\circ}$ to $140^{\circ}$ in $10^{\circ}$ increments, for $2 \min (4 \mathrm{~min}$ at the highest conc.) at each angle. The solvodynamic diameter in the concentrated dispersions was inferred from a first order cumulant fit to the cross-correlation function. We refrain from reporting further details of the particle size distribution, which we believe cannot be determined reliably from the obtained scattering data.

\section{S3. Interfacial Tension Measurement}

Effective interfacial tensions of the hexane-based particle dispersions with water were measured to test the interfacial activity of the Janus snowman particles. We used a Ramé-hart goniometer model250 to carry out video image edge tracing of a pendant drop of oil at an inverted steel needle submerged in the surrounding aqueous phase. The interfacial tension between two immiscible phases was calculated 
based on the shape of the pendant drop near its apex. Measurements were taken for longer than 20 min to measure the interfacial tension at equilibrium with particles saturated at the interface. The time-dependent interfacial tension from which the plateau value is obtained is shown in Figure S2.

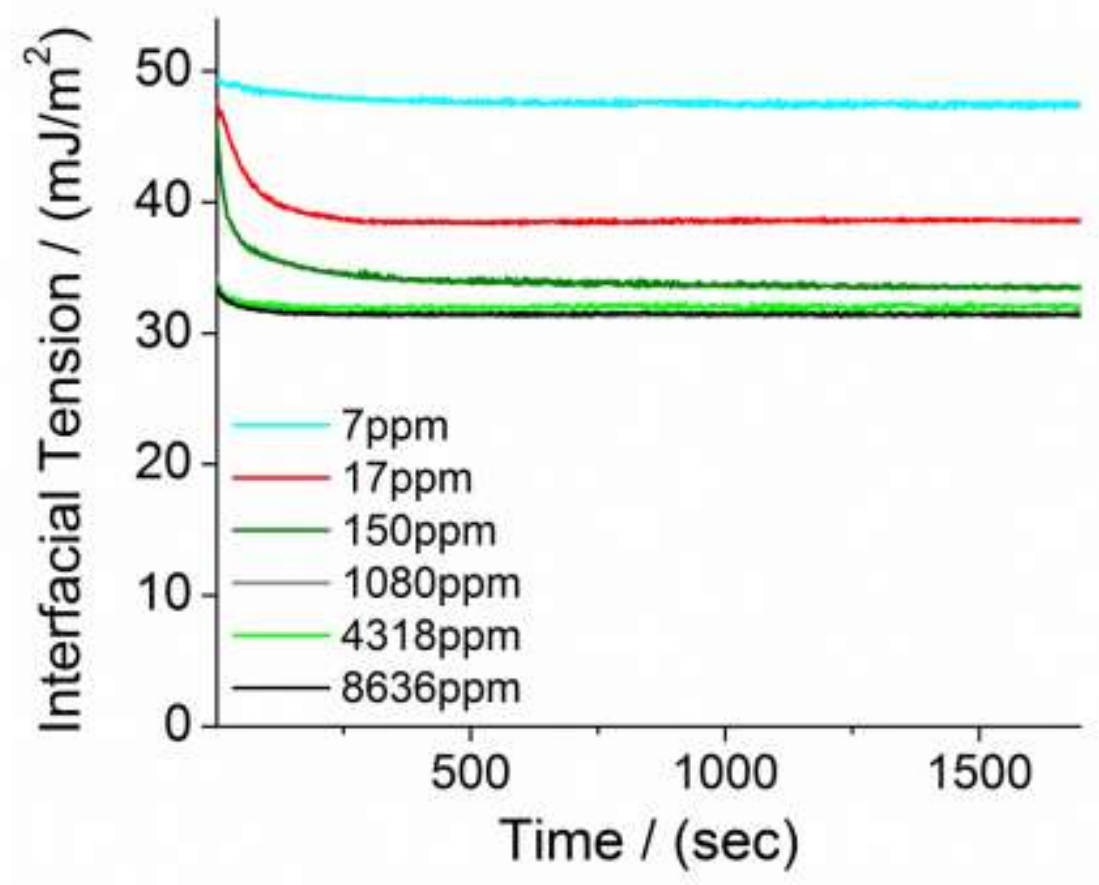

Figure S2. Time-dependent interfacial tension of water with hexane-based dispersions of Janus snowman particles, from which the plateau value (equilibrium interfacial tension) is obtained and shown in Figure $2 \mathrm{~A}$ of the main text.

\section{S4. Conductivity Measurement}

The electric conductivity of hexane containing Janus snowman particles was measured using the nonaqueous conductivity probe DT-700 (Dispersion Technology, Inc.). The probe with coaxial cylindrical electrodes was immersed in the hexane dispersion and an AC field with a low frequency $(1 \mathrm{~Hz})$ is applied between the electrodes during the measurement. The measured current is shown in the form of the specific conductivity based on the cell constant for the given electrode geometry. We confirmed the 
conductivity below $10 \mathrm{pS} / \mathrm{m}$ for pure hexane. ${ }^{2}$ Prior to the measurements, the probe was rinsed with chloroform, wiped in hot aqueous detergent solution, rinsed copiously, first with hot water and then with acetone and methanol, air-dried, stabilized in a fume hood overnight, and finally rinsed with pure hexane.

\section{S5. Electrochemical Impedance Spectroscopy (EIS)}

The electrochemical impedance spectroscopy (EIS) was performed for a dodecane-based dispersion sample of the Janus particles, following the procedure described in the literature. ${ }^{3}$ The sample was sandwiched between two planar electrodes of glass coated with $100 \mathrm{~nm}$ thick layer of indium tin oxide (ITO, Sigma-Aldrich), kept apart by a $250 \mu \mathrm{m}$ polycarbonate spacer. A VersaSTAT 3 potentiostat (Princeton Applied Research), equipped with a Low Current Interface (LCI), powered the cell, a two electrode circuit, by short-circuiting the reference electrode terminal of the potentiostat to the terminal of the counter electrode. The Nyquist plot of the measured real and imaginary impedance is shown in Figure S2A and the frequency response of the real and imaginary part is shown in Figure S2B and S2C. We note that the shape of spectra is qualitatively similar to those for nonpolar solutions of the commercial surfactant product OLOA11000, where the measured electric current was attributed to the electrophoretic motion of charge carrier inverse micelles in the capacitor cell. ${ }^{3}$ The measured fluid resistance $\left(R_{f}\right)$ within the capacitor, for the dodecane sample with the highest particle volume fraction (equivalent to $30303 \mathrm{ppm}$ in mass for the hexane-based sample, shown in Figure 3), was converted into the conductivity $(\sigma)$ by $\sigma=\mathrm{d} / A_{f} R_{f}$ with $\mathrm{d}$ for the distance between two electrodes and $A_{f}$ for the area of the two plates wet by the fluid. The calculated $\sigma$ was $500 \pm 30 \mathrm{pS} / \mathrm{m}$, which is in the same order of magnitude as the measured $\sigma$ using the conductivity probe DT-700 (introduced in S4). The impedance in the frequency range of $10^{0}-10^{-2} \mathrm{~Hz}$ shows evidence of an electrical double layer formed at either electrode. ${ }^{3}$ The capacitance of the double layer could not be used for further characterization of the charge due to the nonnegligible size of the charge carriers. 

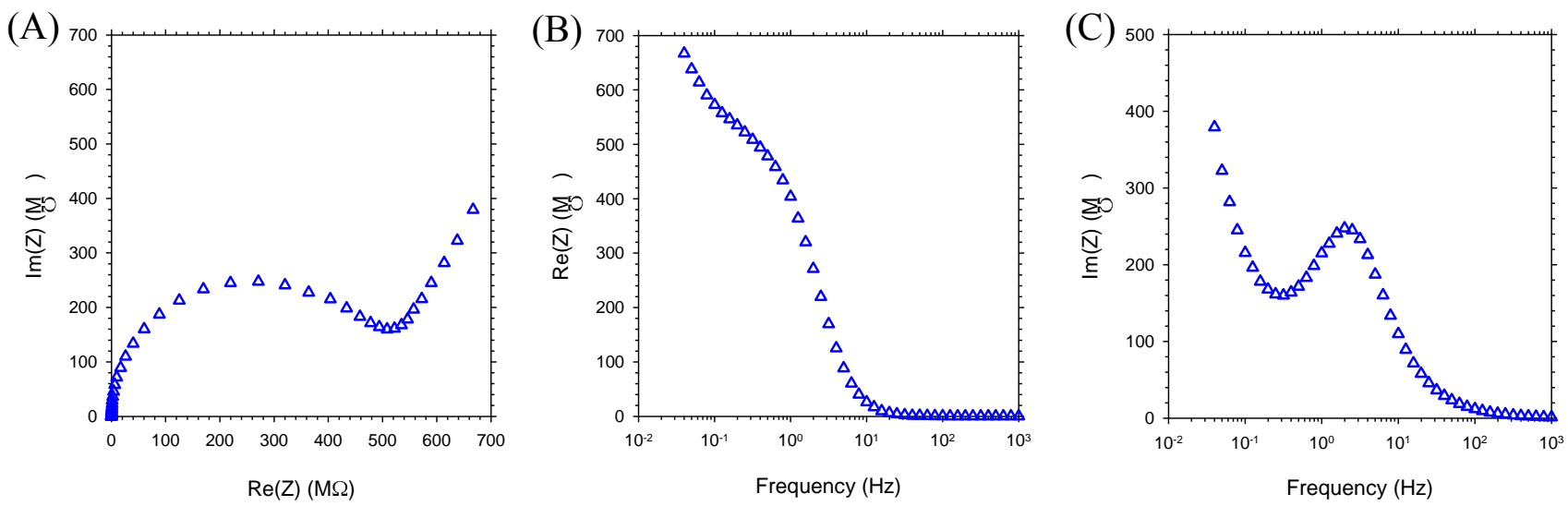

Figure S3. (A) Nyquist plot of impedance data (open symbols) and best fit model (solid line)for the dodecane sample containing the Janus snowman particles. The data was taken at frequencies from $10^{3} \mathrm{~Hz}$ to $10^{-2} \mathrm{~Hz}$. (B) Semi-log Bode plot of the real impedance. (C) Semi-log Bode plot of the imaginary impedance.

\section{S6. Karl Fischer Titration.}

The moisture content in nonpolar dispersions was determined by volumetric Karl Fischer titration using TitroLine KF titrator (SCHOTT). HYDRANAL® - Composite 5 (Sigma Aldrich) was used as a titration reagent and methanol dry (Sigma Aldrich) was used as a titration medium. We confirmed a water content $\sim 30 \mathrm{ppm}$ for pure hexane, consistent with earlier findings. ${ }^{2}$

\section{REFERENCES}

(1) Park, J.-G.; Forster, J. D.; Dufresne, E. R. J. Am. Chem. Soc. 2010, 132, 5960.

(2) Guo, Q.; Singh, V.; Behrens, S. H. Langmuir 2010, 26, 3203.

(3) Yezer, B. A.; Khair, A. S.; Sides, P. J.; Prieve, D. C. J. Colloid Interface Sci. 2015, 449, 2. 